\title{
Michael J.C. Gordon FRS Professor of Computer Assisted Reasoning (28 February 1948-22 August 2017)
}

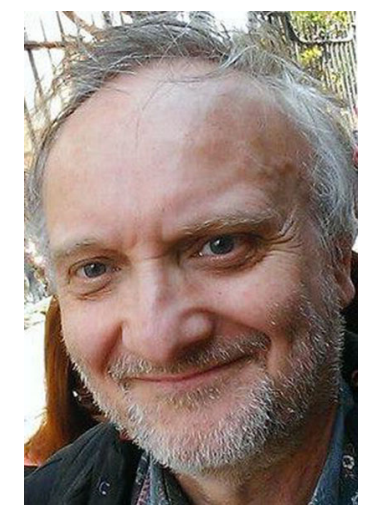

Professor Michael Gordon was a pioneer in the field of interactive theorem proving, with a focus on hardware verification. This field is concerned with certifying system designs by proving their correctness mathematically. Mike Gordon shaped this field from the beginning, demonstrating the feasibility of hardware verification on realworld computer designs. His students extended the work to such diverse areas as the verification of floating-point algorithms, the verification of probabilistic algorithms and the verified translation of source code to (necessarily correct) machine language code. In recognition of his achievements, he was elected to the Royal Society in 1994, and he continued to make valuable contributions until the end of his career.

In the 1970s, as a postdoctoral researcher at Edinburgh University, Mike Gordon was part of the team that built Edinburgh LCF. This was an interactive theorem prover: a program for undertaking formal proofs in a logical calculus (the Logic for Computable Functions). And it was the first of its kind. Although the LCF calculus soon fell out of favour, the architecture of Edinburgh LCF is now almost universally adopted by today's interactive provers. This early project also introduced the ML family of functional programming languages.

Mike met his wife Avra during his first post-doc in 1974, a year spent with John McCarthy at the Stanford Artificial Intelligence Lab where Avra was a Research Assistant. They were colleagues at Edinburgh and Cambridge until Avra retired in 1991 to raise the family.

Mike Gordon was appointed to a Lectureship at Cambridge in 1981. There he turned his attention to hardware, introducing first LCF_LSM (Logic for Sequential Machines) and then HOL (Higher Order Logic). One of his key contributions was to demonstrate the effectiveness of higher order logic as a general formalism for verification, replacing earlier specialised formalisms. At the time, first order logic was preferred both by logicians themselves and by the AI community; Mike demonstrated that higher order logic could be implemented effectively and used to specify hardware designs from the gate level right up to the processor level, as well as abstract hardware specifications. A steady stream of $\mathrm{PhD}$ students extended the applicability and power of the HOL system to unimagined levels. Cambridge promoted Mike to Reader in 1988 and Professor in 1996.

The impact of his work, along with that of the students and colleagues, is worldwide. Techniques that originated in his group at Cambridge are used by major chip vendors and have deeply influenced the entire field of interactive theorem proving.

Mike Gordon's colleagues and students will remember him as an attentive and supportive listener, of unfailing kindness and generosity. He is survived by his wife, Avra Cohn, and by their two children Katriel and Reuben Cohn-Gordon. 